\title{
Undetermined coefficients for local fractional differential equations
}

\author{
Roshdi Khalila,*, Mohammed Al Horani ${ }^{a, b}$, Douglas Anderson ${ }^{c}$ \\ ${ }^{a}$ Department of Mathematics, The University of Jordan, Amman, Jordan. \\ ${ }^{b}$ Department of Mathematics, Faculty of Science, University of Hail, Saudi Arabia. \\ ${ }^{c}$ Department of Mathematics, Concordia College, Moorhead, MN, USA.
}

\begin{abstract}
We discuss the method of undetermined coefficients for fractional differential equations, where we use the (local) conformable fractional derivative presented in [R. Khalil, M. Al Horani, A. Yousef, M. Sababheh, J. Comput. Appl. Math., 264 (2014), 65-70]. The concept of fractional polynomials, fractional exponentials and fractional trigonometric functions is introduced. A method similar to the case of ordinary differential equations is established to find a particular solution for nonhomogenous linear fractional differential equations. Some other results are presented. (c)2016 All rights reserved.

Keywords: Conformable fractional, derivative, fractional integral, fractional differential equation, undetermined coefficients.
\end{abstract}

$2010 M S C: 26 \mathrm{~A} 33$.

\section{Introduction}

Fractional differential equations have been of great interest for the last thirty years because of their applications in applied sciences, see [6], [8] and [12]. The main definitions which are of wide use are the Riemann-Liouville definition and the Caputo definition, see [10, 11].

\section{*Corresponding author}

Email addresses: roshdi@ju.edu.jo (Roshdi Khalil), horani@ju.edu.jo, m.alhorani@uoh.edu.sa (Mohammed Al Horani), andersod@cord.edu (Douglas Anderson) 
(i) Riemann - Liouville Definition. For $\alpha \in[n-1, n)$, the $\alpha$ derivative of $f$ is

$$
D_{a}^{\alpha}(f)(t)=\frac{1}{\Gamma(n-\alpha)} \frac{d^{n}}{d t^{n}} \int_{a}^{t} \frac{f(x)}{(t-x)^{\alpha-n+1}} d x .
$$

(ii) Caputo Definition. For $\alpha \in[n-1, n)$, the $\alpha$ derivative of $f$ is

$$
D_{a}^{\alpha}(f)(t)=\frac{1}{\Gamma(n-\alpha)} \int_{a}^{t} \frac{f^{(n)}(x)}{(t-x)^{\alpha-n+1}} d x .
$$

However, the following are some of the setbacks of one definition or the other:

(i) The Riemann-Liouville derivative does not satisfy $D_{a}^{\alpha}(1)=0\left(D_{a}^{\alpha}(1)=0\right.$ for the Caputo derivative), if $\alpha$ is not a natural number.

(ii) All fractional derivatives do not satisfy the known formula of the derivative of the product of two functions:

$$
D_{a}^{\alpha}(f g)=f D_{a}^{\alpha}(g)+g D_{a}^{\alpha}(f) .
$$

(iii) All fractional derivatives do not satisfy the known formula of the derivative of the quotient of two functions:

$$
D_{a}^{\alpha}(f / g)=\frac{g D_{a}^{\alpha}(f)-f D_{a}^{\alpha}(g)}{g^{2}} .
$$

(iv) All fractional derivatives do not satisfy the chain rule:

$$
D_{a}^{\alpha}(f \circ g)(t)=f^{(\alpha)}(g(t)) g^{(\alpha)}(t) .
$$

(v) All fractional derivatives do not satisfy: $D^{\alpha} D^{\beta} f=D^{\alpha+\beta} f$, in general.

(vi) All fractional derivatives, especially the Caputo definition, assume that the function $f$ is differentiable.

In [9], the authors gave a new definition of a (local) fractional derivative which is a natural extension to the usual first derivative as follows:

Let $f:[0, \infty) \longrightarrow \mathbb{R}$ be a given function. Then for all $t>0$ and $\alpha \in(0,1)$, define $T_{\alpha}(f)$ via

$$
T_{\alpha}(f)(t)=\lim _{\varepsilon \rightarrow 0} \frac{f\left(t+\varepsilon t^{1-\alpha}\right)-f(t)}{\varepsilon} .
$$

$T_{\alpha}$ is called the conformable fractional derivative of $f$ of order $\alpha$.

Let $f^{(\alpha)}(t)$ stand for $T_{\alpha}(f)(t)$. It then follows that

$$
f^{(\alpha)}(t)=\lim _{\varepsilon \rightarrow 0} \frac{f\left(t+\varepsilon t^{1-\alpha}\right)-f(t)}{\varepsilon} .
$$


If $f$ is $\alpha$-differentiable in some $(0, b), b>0$, and $\lim _{t \rightarrow 0^{+}} f^{(\alpha)}(t)$ exists, then define

$$
f^{(\alpha)}(0)=\lim _{t \rightarrow 0^{+}} f^{(\alpha)}(t) .
$$

According to this definition, the following statements are true, see [9], see also [1, 2, 3, 4, 5, 17,

1. $T_{\alpha}\left(t^{p}\right)=p t^{p-\alpha}$ for all $p \in \mathbb{R}$.

2. $T_{\alpha}\left(\sin \frac{1}{\alpha} t^{\alpha}\right)=\cos \frac{1}{\alpha} t^{\alpha}$.

3. $T_{\alpha}\left(\cos \frac{1}{\alpha} t^{\alpha}\right)=-\sin \frac{1}{\alpha} t^{\alpha}$.

4. $T_{\alpha}\left(e^{\frac{1}{\alpha} t^{\alpha}}\right)=e^{\frac{1}{\alpha} t^{\alpha}}$.

Further, all the classical properties of the derivative hold. This suggests that one may try to solve (local) fractional differential equations using the same techniques for solving ordinary differential equations. It is the purpose of this paper to study the method of undetermined coefficients to find particular solutions for linear fractional differential equations. The concept of fractional polynomials, fractional exponential, and fractional trigonometric functions are introduced, and then applied for the undetermined coefficients method.

\section{Fractional Polynomial Functions}

Let $X$ be a vector space, and $T: X \rightarrow X$, be a linear operator on $X$. A subspace $M \subset X$, is called invariant under $T$, if $T(M) \subseteq M$. Such a concept was used in the method of undetermined coefficients for ordinary differential equations. For such a situation, $T$ was the differential operator, and $M$ was taken to be the space generated by polynomials, exponential functions, and trigonometric functions, added or multiplied.

Definition 2.1. Let $n \in \mathbb{N}$, the set of natural numbers, and $\alpha \in(0,1)$. We call $\alpha$ a factor of $n$ if there exists $k \in \mathbb{N}$ such that $k \alpha=n$. of 1 .

For example, $\frac{1}{2}$ is a factor of 2 with $k=4$, and $\frac{1}{3}$ is a factor of 1 with $k=3$. But $\frac{3}{5}$ is not a factor

Definition 2.2. A fractional polynomial of degree $n$ and factor $\alpha$ is a function of the form

$$
P(x)=a_{n} x^{n}+a_{n-1} x^{n-\alpha}+\ldots . .+a_{n-k-1} x^{\alpha}+a_{n-k},
$$

where $a_{j} \in \mathbb{R}$, the set of real numbers. We write $P(x)$ is an $(n, \alpha)-$ fractional polynomial. If $a_{n}=0$, we take $n$ to be the smallest $n$ for which $\alpha$ is a factor.

For example, $x+x^{\frac{1}{2}}-4$ is a $\left(1, \frac{1}{2}\right)$-fractional polynomial, and $2 x^{\frac{3}{2}}+5 x-x^{\frac{1}{2}}+7$ is a $\left(2, \frac{1}{2}\right)$-fractional polynomial. In addition, $x+x^{\frac{2}{3}}-1$ is a $\left(1, \frac{1}{3}\right)$-fractional polynomial. Here the coefficient of $x^{\frac{1}{3}}$ is 0 .

Let $J(\alpha)$ be the set of all $(n, \alpha)$-polynomials for all $n \in \mathbb{N}$ and fixed $\alpha$; clearly, $J(\alpha)$ is a subspace of the space of all continuous functions on $[0, \infty)$. Let $G(\alpha)$ be the space of all functions of the form $c_{1} \sin \left(\frac{t^{\alpha}}{\alpha}\right)+c_{2} \cos \left(\frac{t^{\alpha}}{\alpha}\right)$, where $c_{1}, c_{2} \in \mathbb{R} ; E(\alpha)$ be the space of all functions of the form $c e^{\frac{t^{\alpha}}{\alpha}}$, where $c \in \mathbb{R}$; and let $M(\alpha)$ be the space of all functions of the form $e^{\frac{t^{\alpha}}{\alpha}}\left(P_{1}(t) \sin \left(\frac{t^{\alpha}}{\alpha}\right)+P_{2}(t) \cos \left(\frac{t^{\alpha}}{\alpha}\right)\right)$, where $P_{1}, P_{2} \in J(\alpha)$. 
Theorem 2.3. The subspaces $J(\alpha), G(\alpha), E(\alpha)$, and $M(\alpha)$ are invariant under the linear map $T_{\alpha}$.

Proof. Using Theorem 2.2 in [9], and the facts, see also [9],

$$
\begin{aligned}
& T_{\alpha}\left(e^{\frac{1}{\alpha} t^{\alpha}}\right)=e^{\frac{1}{\alpha} t^{\alpha}} \\
& T_{\alpha}\left(\sin \frac{1}{\alpha} t^{\alpha}\right)=\cos \left(\frac{1}{\alpha} t^{\alpha}\right), \\
& T_{\alpha}\left(\cos \frac{1}{\alpha} t^{\alpha}\right)=-\sin \left(\frac{1}{\alpha} t^{\alpha}\right), \\
& T_{\alpha}\left(t^{p}\right)=p t^{p-\alpha}
\end{aligned}
$$

the result follows.

We should remark that the spaces $J(1), G(1), E(1)$, and $M(1)$ are invariant under $T_{1}$, which is the first derivative.

\section{Method of Undetermined Coefficients}

Let us start with the following definition.

Definition 3.1. A differential equation is called an $(n, \alpha)$ - fractional differential equation if it is of the form

$$
a_{n} y^{(n)}+a_{n-1} y^{(n-\alpha)}+\ldots+a_{n-k-1} y^{(\alpha)}+a_{n-k} y=f(t),
$$

where $\alpha$ is a factor of the natural number $n$. If $a_{n}=0$, we take $n$ to be the smallest $n$ for which $\alpha$ is a factor. The differential equation (3.1) is called a fractional differential equation of order $n$ and factor $\alpha$.

Since $T_{\alpha}$ is linear, see [9], Theorem 2.2, then one can easily see that equation (3.1) is linear.

In this section we consider equations of the form

$$
y^{(\alpha)}+a y=f(t)
$$

where $f(t)$ is an element of one of the spaces $J(\alpha), G(\alpha), E(\alpha)$, and $M(\alpha)$.

Let us write $y_{h}$ for the solution of the homogenous equation $y^{(\alpha)}+a y=0$, and $y_{p}$ for any particular solution of $y^{(\alpha)}+a y=f(t)$. Then as in the case of ordinary differential equations, the general solution is $y_{g}=y_{h}+y_{p}$.

The equation $y^{(\alpha)}+a y=0$ can be written as $\left(T_{\alpha}+a I\right) y=0$, where $I$ is the identity operator on the space of continuous functions on $[0, \infty)$. Hence, $y_{h}$ is an element of the kernel of the operator $T_{\alpha}+a I$.

Now, since $T_{\alpha}\left(e^{\frac{1}{\alpha} t^{\alpha}}\right)=e^{\frac{1}{\alpha} t^{\alpha}}$, it follows that $T_{\alpha}\left(e^{\frac{-a}{\alpha} t^{\alpha}}\right)=-a e^{\frac{-a}{\alpha} t^{\alpha}}$. It follows that the kernel of $T_{\alpha}+a I$ consists of the functions $b e^{\frac{-a}{\alpha} t^{\alpha}}$, for $b \in \mathbb{R}$. Thus $y_{h}$ for $y^{(\alpha)}+a y=0$ is $y_{h}=b e^{\frac{-a}{\alpha} t^{\alpha}}$.

Remark 3.2. It is interesting to observe that the general solution of the homogenous part of equation (3.2) is $y_{h}=e^{r \frac{1}{\alpha} t^{\alpha}}$ and to notice that one can form the auxiliary equation $r+a=0$, where $y^{(\alpha)}$ is replaced by $r$. So $r=-a$, and $y_{h}=e^{-a \frac{1}{\alpha} t^{\alpha}}$. For example, the equation $y^{\left(\frac{1}{2}\right)}-y=0$, has auxiliary equation $r-1=0$, so $r=1$, and hence $y_{h}=e^{2 \sqrt{t}}$.

Now, to find $y_{p}$ using the method of undetermined coefficients, the function $f(t)$ must be in one of the spaces $J(\alpha), G(\alpha), E(\alpha)$, and $M(\alpha)$. This is because such spaces are invariant under $T_{\alpha}$, and hence under $T_{\alpha}+a I$. 
Consequently, if $f(t)=c e^{t^{\alpha}}$, then $y_{p}$ must be $b e^{t^{\alpha}}$, where $b$ is to be determined by substituting $b e^{t^{\alpha}}$ in $y^{(\alpha)}+a y=c e^{t^{\alpha}}$.

If $f(t)=c_{1} \sin t^{\alpha}+c_{2} \cos t^{\alpha}$, then $y_{p}$ must be $b_{1} \sin t^{\alpha}+b_{2} \cos t^{\alpha}$, where $b_{1}$ and $b_{2}$ are to be determined by substituting in the equation $y^{(\alpha)}+a y=c_{1} \sin t^{\alpha}+c_{2} \cos t^{\alpha}$.

Similarly if $f(t)$ is an $(n, \alpha)$-fractional polynomial.

Remark 3.3. The above discussion works for the form of $y_{p}$ as long as there is no similarity between $y_{h}$ and $f(t)$. In such a case, we have to modify the form of $y_{p}$ as we will show later in this paper.

Example 3.4. Let us consider the general solution of the following fractional differential equations:

(1) $y^{\left(\frac{1}{2}\right)}+2 y=\sin \sqrt{t}$.

Solution. The auxiliary equation of $y^{\left(\frac{1}{2}\right)}+2 y=0$ is $r+2=0$, so $r=-2$. Hence $y_{h}=b e^{-2(2) \sqrt{t}}=$ $b e^{-4 \sqrt{t}}$.

Now, $y_{p}=A \sin \sqrt{t}+B \cos \sqrt{t}$, noting there is no similarity between $y_{h}$ and any of the terms of $y_{p}$. Substituting $y_{p}$ in the equation $y^{\left(\frac{1}{2}\right)}+2 y=\sin \sqrt{t}$, we get $A=\frac{8}{17}$ and $B=-\frac{2}{17}$. Hence

$$
y_{g}=b e^{-4 \sqrt{t}}+\frac{8}{17} \sin \sqrt{t}-\frac{2}{17} \cos \sqrt{t} \text {. }
$$

(2) $y^{\left(\frac{1}{3}\right)}-y=t$.

Solution. The auxiliary equation of $y^{\left(\frac{1}{3}\right)}-y=0$ is $r-1=0$, so $r=1$. Hence

$$
y_{h}=b e^{3 \sqrt[3]{t}}
$$

Again, $y_{p}=a t+b t^{\frac{2}{3}}+c t^{\frac{1}{3}}+d$, since there is no similarity between any of the terms of $y_{p}$ and $y_{h}$. Substituting $y_{p}$ in the equation $y^{\left(\frac{1}{3}\right)}-y=t$, we get

$$
a=-1, b=1, c=\frac{2}{3}, d=\frac{2}{9} .
$$

(3) $y^{\left(\frac{1}{2}\right)}-3 y=t e^{\sqrt{t}} \cos \sqrt{t}$.

Solution. One can easily see that $y_{h}=b e^{6 \sqrt{t}}$. As for $y_{p}$, the form is

$$
y_{p}=e^{\sqrt{t}}\left(c_{1} t+c_{2} \sqrt{t}+c_{3}\right)(A \sin \sqrt{t}+B \cos \sqrt{t}),
$$

noting that there is no similarity between $y_{h}$ and any of the terms of $y_{p}$.

So just substituting $y_{p}$ in our equation and determine the coefficients.

\section{The case of similarity}

What if there is a similarity between $y_{h}$ and any of the terms of $y_{p}$ ? Here is such an example

$$
y^{\left(\frac{1}{2}\right)}-y=5 e^{2 \sqrt{t}} .
$$


Here $y_{h}=e^{2 \sqrt{t}}$, and the form of $y_{p}$ is $y_{p}=b e^{2 \sqrt{t}}$. But if we substitute $y_{p}$ in equation (4.1), we will not be able to determine $b$. Hence we try $y_{p}=b \sqrt{t} e^{2 \sqrt{t}}$. Substitute such $y_{p}$ in equation 4.1 to get

$$
b \sqrt{t} e^{2 \sqrt{t}}+\frac{1}{2} b e^{2 \sqrt{t}}-b \sqrt{t} e^{2 \sqrt{t}}=5 e^{2 \sqrt{t}}
$$

Hence $b=10$. So $y_{g}=c e^{2 \sqrt{t}}+10 \sqrt{t} e^{2 \sqrt{t}}$.

Thus in case of similarity between any of the terms of $y_{p}$ and $y_{h}$ we multiply the assumed form of $y_{p}$ by $t^{\alpha}$, whenever the equation is $y^{(\alpha)}+b y=f(t)$.

Open Problem 1. Is there an auxiliary equation for equations

$$
a_{n} y^{(n)}+a_{n-1} y^{(n-\alpha)}+\ldots+a_{n-k-1} y^{(\alpha)}+a_{n-k} y=f(t),
$$

with at least one coefficient of some derivative(not fractional) not equal to zero? As an example $y^{\prime \prime}+y^{\left(\frac{3}{2}\right)}-y=0 ?$

Open Problem 2. If $f(t)$ does not belong to any of the spaces $J(\alpha), G(\alpha), E(\alpha)$, and $M(\alpha)$, is there a method of undetermined coefficients to find $y_{p}$ ?

For example, in the case of (3.2), we have the following affirmative result.

Lemma 4.1. Let $f, a:\left[t_{0}, \infty\right) \subset[0, \infty)$ be continuous, and let $y_{0} \in \mathbb{R}$. Then the unique solution of the initial value problem

$$
y^{(\alpha)}(t)+a(t) y(t)=f(t), \quad y\left(t_{0}\right)=y_{0},
$$

is given by

$$
y(t)=y_{0} e^{-\int_{t_{0}}^{t} a(\tau) \tau^{\alpha-1} d \tau}+\int_{t_{0}}^{t} e^{-\int_{s}^{t} a(\tau) \tau^{\alpha-1} d \tau} f(s) s^{\alpha-1} d s, \quad t \in\left[t_{0}, \infty\right)
$$

Proof. Let $y$ be given by $(4.2)$. Using the conformable fractional derivative rules,

$$
\begin{aligned}
D^{\alpha} y(t) & =-y_{0} a(t) e^{-\int_{t_{0}}^{t} a(\tau) \tau^{\alpha-1} d \tau}+f(t) e^{-\int_{t}^{t} a(\tau) \tau^{\alpha-1} d \tau}-\int_{t_{0}}^{t} a(t) e^{-\int_{s}^{t} a(\tau) \tau^{\alpha-1} d \tau} f(s) s^{\alpha-1} d s \\
& =-a(t) y(t)+f(t)
\end{aligned}
$$

which completes the proof of the lemma.

\section{References}

[1] T. Abdeljawad, On conformable fractional calculus, J. Comput. Appl. Math., 279 (2015), 57-66. 1

[2] T. Abdeljawad, M. Al Horani, R. Khalil, Conformable Fractional Semigroups of Operators, J. Semigroup Theory Appl., 2015 (2015), 11 pages. 1

[3] M. Abu Hammad, R. Khalil, Abel's formula and Wronskian for conformable fractional differential equations, Int. J. Differential Equations Appl., 13 (2014), 177-183.11

[4] B. Bayour, D. F. M. Torres, Existence of solution to a local fractional nonlinear differential equation, J. Comput. Appl. Math., (2016), (in press). 1

[5] N. Benkhettou, S. Hassani, D. F. M. Torres, A conformable fractional calculus on arbitrary time scales, J. King Saud Univ., 28 (2016), 93-98.1

[6] T. Caraballoa, M. Abdoul Diopb, A. A. Ndiayeb, Asymptotic behavior of neutral stochastic partial functional integro-differential equations driven by a fractional Brownian motion, J. Nonlinear Sci. Appl., 7 (2014), 407-421.1. 
[7] A. Gokdogan, E. Unal, E. Celik, Existence and Uniqueness Theorems for Sequential Linear Conformable Fractional Differential Equations, to appear in Miskolc Mathematical Notes. 1

[8] M. Hao, C. Zhai, Application of Schauder fixed point theorem to a coupled system of differential equations of fractional order, J. Nonlinear Sci. Appl., 7 (2014), 131-137.11

[9] R. Khalil, M. Al Horani, A. Yousef, M. Sababheh, A new Definition of Fractional Derivative, J. Comput. Appl. Math., 264 (2014), 65-70.1, 2, 3

[10] A. Kilbas, H. Srivastava, J. Trujillo, Theory and applications of fractional differential equations, Math. Studies. Northholland, NewYork, (2006). 1

[11] K. S. Miller, An introduction to fractional calculus and fractional differential equations, J.Wiley and Sons, New York, (1993).1

[12] J. A. Nanware, D. B. Dhaigude, Existence and uniqueness of solutions of differential equations of fractional order with integral boundary conditions, J. Nonlinear Sci. Appl., 7 (2014), 246-254.1 